\title{
Lidar and Electro-Optics for Atmospheric Hazard Sensing and Mitigation
}

\author{
Ivan O. Clark * \\ NASA Langley Research Center, Hampton, VA 23681
}

\begin{abstract}
This paper provides an overview of the research and development efforts of the Lidar and Electro-Optics element of NASA's Aviation Safety Program. This element is seeking to improve the understanding of the atmospheric environments encountered by aviation and to provide enhanced situation awareness for atmospheric hazards. The improved understanding of atmospheric conditions is specifically to develop sensor signatures for atmospheric hazards. The current emphasis is on kinetic air hazards such as turbulence, aircraft wake vortices, mountain rotors, and windshear. Additional efforts are underway to identify and quantify the hazards arising from multi-phase atmospheric conditions including liquid and solid hydrometeors and volcanic ash. When the multi-phase conditions act as obscurants that result in reduced visual awareness, the element seeks to mitigate the hazards associated with these diminished visual environments. The overall purpose of these efforts is to enable safety improvements for air transport class and business jet class aircraft as the transition to the Next Generation Air Transportation System occurs.
\end{abstract}

\section{Introduction}

$\mathrm{T}$ HE Atmospheric Environment Safety Technologies (AEST) Project is one of the three projects that make up the NASA Aviation Safety Program. It investigates sources of risk and provides technology needed to help ensure safe flight in and around atmospheric hazards. This is done in the context of the current National Air Transportation System and with the goal of maintaining or increasing safety as the transition to the Next Generation Air Transportation System (NextGen) $)^{1}$ occurs. The AEST Project is structured as three sub-projects. Each of these subprojects is implemented through technical work elements. The Lidar and Electro-Optic (LEO) Element is part of the Atmospheric Hazard Sensing and Mitigation (AHSM) Sub-Project. The approach of the LEO Element is to explore the capabilities of evolving sensor technologies to monitor the atmospheric environment from aircraft and to detect and assess atmospheric hazards so as to allow avoidance or mitigation of those hazards. The goal is to enable future avionics systems with multi-hazard sensing and mitigation capabilities that will provide improved safety of flight for air transport class aircraft as well as for smaller aircraft such as those in the business jet class.

The LEO Element is investigating both passive and active electro-optic sensor systems for sensing and assessing atmospheric hazards. This involves both determining the sensor signature for a given pair of atmospheric hazard and sensor type and evaluating whether the current state of technology for that sensor type allows quantitative measurement of the sensor signature. Part of this evaluation explores the signal-to-noise ratio required by the sensor to minimize both false positives and false negatives for hazard assessment. In many cases, the atmospheric conditions and structures that create hazards to aviation are not fully understood and LEO seeks partners in other NASA programs, in other government programs, in industry, and in academia to cost-effectively develop these understandings in a timely fashion. Because of the emphasis on evolving technologies, LEO efforts are connected to the innovative technology efforts being done under the NASA Small Business Innovative Research (SBIR) program and through cooperative research agreements developed competitively through the NASA Research Announcement (NRA) process.

\section{Passive Sensors}

LEO is investigating hyper-spectral imaging for passive measurement of aircraft wake vortices, mountain waves, turbulence, diminished visual environment operations, and runway surface state. This investigation utilizes a

\footnotetext{
*AeroSpace Technologist - AEST AHSM LEO Element Lead, Electromagnetics \& Sensors Branch, Mail Stop 473.
} 
forward-looking interferometric (FLI) imaging system. ${ }^{2}$ This effort is directly examining the capabilities of state-oftechnology interferometric imagers for aviation safety applications. It is also seeking to identify optical wavelengths that could be used for multi-spectral imagers for these applications. A field experiment was conducted at the University of Colorado Boulder's Mountain Research Station with a current state-of-technology FLI in November 2011. ${ }^{3}$ It emphasized measurements for orographic turbulence due to mountain waves. A secondary emphasis was on surface-state measurements relevant to water, snow, and ice on runways. The analysis of the data from this field experiment is in progress. This effort is primarily funded through a NRA and conducted by a consortium including Hampton University and Starr Institute and led by Georgia Tech Research Institute with collaboration by Ohio University and Telops.

In-house numerical modeling of hyper-spectral imaging of wake vortices has suggested that a 6-band multispectral imager would be able to detect and assess the hazard level from en route wake vortex encounters. ${ }^{4}$ This is a recent development and is expected to be the focus of LEO efforts in passive imaging in fiscal year 2013 and beyond.

\section{Active Sensors}

LEO is pursuing multiple lidar and active imaging approaches for atmospheric hazards through both in-house and contractual efforts.

A coherent Doppler lidar is being developed by Fibertek, Inc., under a NASA SBIR for airborne detection of wake vortices generated by the aircraft ahead of the plane carrying the lidar. NASA is expecting delivery of this small, compact, fiber laser system late in fiscal year 2012 with in-house testing to follow upon delivery. Initial testing will focus on wind field measurements for wake vortex detection and for turbulence mitigation and on validation of numerical models developed in-house.

Two different approaches to direct Doppler lidar for optical air data systems (OADS) are being evaluated by Michigan Aerospace Corporation and by Ophir Corporation under separate NASA SBIR efforts. OADS have the potential to provide 3D winds ahead as well as temperature and pressure density. This class of sensor has the potential to enable both turbulence mitigation and independent validation of the current pitot tube and vane instruments.

A recently concluded NASA SBIR effort with Physical Sciences Inc. showed that a multi-wavelength direct lidar has excellent potential to provide both airborne volcanic ash hazard detection and an in-flight icing hazard warning system.

Active monochromatic imaging using laser illumination is being evaluated for Laser Imaging Through Obscurants (LITO) to mitigate hazards due to diminished visual environments. RL Associates is developing this capability under a NASA SBIR follow-on. Field tests conducted this winter at the Picatinny Arsenal in New Jersey showed excellent promise of mitigating the effects of reduced visibility. Analysis of the test data and correlation with the arsenal's meteorological measurements including disdrometers is in progress. This sensor technology also shows promise for discriminating hazards that can arise from the obscurants themselves such as icing.

\section{Hazards}

The current LEO emphasis is on kinetic air hazards such as turbulence, aircraft wake vortices, mountain rotors, and windshear. Of these, the relatively well-defined structure and limited spatial extent of aircraft wake vortices and mountain rotors suggest that avoidance is the best operational paradigm.

The atmospheric conditions experienced by aircraft as turbulence, especially clear air turbulence, can not currently be detected with sufficient range to allow avoidance. In addition, they are often of such large geographic extent that avoidance based on meteorological predictions would result in significant degradation of the NextGen operations. Studies such as those done by Stewart ${ }^{5}$ have shown that modern aircraft have sufficient control authority to significantly mitigate the effects of turbulence on aircraft and their passengers and crew. Enabling this mitigation capability requires high-resolution, accurate measurement of the three dimensional wind field ahead of the aircraft. The current-generation Doppler lidar systems referenced above are very promising to provide these 3D wind measurements. Their size, weight, and power requirements are also being reduced such that they are very promising avionics candidates for turbulence mitigation.

Aircraft wake vortices have traditionally been a hazard in the terminal area for aviation. The mandated minimum spatial separation between aircraft in the terminal area is based on a safety assurance approach to avoid encounters by one aircraft with the wake vortex of an aircraft ahead while the vortex is still strong enough to present a hazard. Some of the proposed NextGen approaches seek to increase capacity by reducing the aircraft separations and maintaining safety levels through measurement of the locations of the wake vortices either by ground-based or 
aircraft-based instruments. In addition, both the 4D separation approach of NextGen and the proposed use of formation flight by civil aviation to improve fuel efficiency result in increased likelihood of hazardous en route wake vortex encounters unless aircraft-based instruments are used for wake avoidance. In addition to the sensor research for wake vortices discussed in the previous section, Aerospace Innovations, LLC, has been selected under a NRA to perform research on sensor signatures for wake vortex detection and hazard assessment. The sensor signatures being considered include both laser and RF radar.

It is still a research question as to whether avoidance, mitigation, or a combination of both is the optimum solution for the types of hazardous windshear and microbursts that are not addressed by current windshear weather radars. Doppler lidar systems and optical air data systems have the potential for addressing these hazards. LEO plans to analyze the range at which these systems would be able to make a hazard assessment as the sensor performance and the sensor numerical models evolve.

The losses of Air France Flight 447 in 2009 and of Northwest Airlines Flight 6231 show the seriousness of loss of situation awareness due to icing of aircraft sensors. By providing non-mechanical measurements of temperature, pressure, and winds ahead; OADS have the potential to provide independent validation of the mechanical aircraft data systems. If the OADS can be developed with sufficient range and fidelity, then it can also serve as the sensor for mitigation turbulence of other kinetic air hazards.

Additional efforts are underway to identify and quantify the hazards arising from multi-phase atmospheric conditions including liquid and solid hydrometeors and volcanic ash. Among the hazards to flight created by multiphase conditions is reduced visual awareness where the condensed phases act as obscurants. As discussed above, the LEO element is investigating technologies to mitigate the hazards associated with these diminished visual environments through passive hyper- or multi-spectral imaging or through active laser imaging through obscurants.

LEO is also collaborating in the Vehicle Integrated Propulsion (VIPR) effort to investigate the effects of volcanic ash ingestion for high-bypass aircraft engines. ${ }^{6}$ This effort seeks to understand the destructive effects of volcanic ash on current production jet engines. ${ }^{7}$

\section{Concluding Remarks}

The Lidar and Electro-Optic Element of the NASA Atmospheric Environment Safety Technologies Project is seeking to enable improvements to aviation safety through evolving electro-optics-based atmospheric sensor systems. The future avionics based on these enabling atmospheric sensor systems will provide cost-effective safety improvements while reducing the impact of weather on aircraft operations in the Next Generation Air Transportation System.

\section{References}

1 Stough, H. Paul III: Aircraft Weather Mitigation for the Next Generation Air Transportation System. American Meteorological Society 87th Annual Meeting - 23rd Conference on Interactive Information and Processing Systems, Paper 9B.8, San Antonio, TX, January 14-18, 2007.

${ }^{2}$ West, L .L.; Gimmestad, G.G.; Smith, W .L.; Kireev, S.; Cornman, L. B.; Schaffner, P. R.; Tsoucalas, G.: Applications of a Forward-Looking Interferometer (FLI) for the On-board Detection of Aviation Weather Hazards. NASA Technical Publication TP-2008-215536, Oct. 2008.

${ }^{3}$ Schaffner, Philip R.; Daniels, Taumi S.; West, Leanne L.; Gimmestad, Gary G.; Lane, Sarah E.; Burdette, Edward M.; Smith, William L.; Kireev, Stanislav; Cornman, Larry; and Sharman Robert D.: "Experimental Validation of a Forward Looking Interferometer for Detection of Clear Air Turbulence due to Mountain Waves," 4th AIAA Atmospheric and Space Environments Conference, New Orleans, LA, June 25-28, 2012.

${ }^{4}$ Daniels, Taumi S.; Smith, William L.; Kireev, Stanislav; "Recent Developments on Airborne Forward Looking Interferometer for the Detection of Wake Vortices," 4th AIAA Atmospheric and Space Environments Conference, New Orleans, LA, June 25-28, 2012.

${ }^{5}$ Stewart, Eric C.: "Turbulence Hazard Metric Based on Peak Accelerations for Jetliner Passengers," NASA TP-2005213528, February 2005.

6 "Aircraft Turbine Engine Volcanic Ash Ingestion Research Testing," International Volcanic Ash Task Force (IVATF), $4^{\text {th }}$ meeting, Montréal, June 13-15, 2012 (to be published).

${ }^{7}$ Creech, Gray, "Jet Engines Eat Cereal and Crayons (No, really)," Armed with Science: Research and Applications for the Modern Military [online journal], May 24, 2012, URL: http://science.dodlive.mil/2012/05/24/jet-engines-eat-cereal-and-crayonsno-really/ [cited 7 June 2012]. 